\title{
Emerging IoT Technologies in Transportation
}

\author{
M. Sundarramurthi \\ Department of Electronics \& Telecommunication \\ Dayananda Sagar College of Engineering(DSCE) \\ Bangalore, India \\ Aravind. R. Krishnan \\ Department of Electronics \& Telecommunication \\ Dayananda Sagar College of Engineering(DSCE) \\ Bangalore, India
}

\author{
Nandita $S$ \\ Department of Electronics \& Telecommunication \\ Dayananda Sagar College Of Engineering(DSCE) \\ Bangalore, India \\ Kruthi Chandrashekar \\ Department of Electronics \& Telecommunication \\ Dayananda Sagar College Of Engineering(DSCE) \\ Bangalore, India
}

\begin{abstract}
This paper focuses primarily on the transportation applications of IoT wherein we discuss how IoT enhances traffic management, accuracy and other such distinct features of traditional transportation systems. The following refers extensively to the potentials, the usefulness, the applications and the maturation of the Internet of Things in the field of transportation and includes suggestions and improvements that can possibly be implemented to the proposed plans.
\end{abstract}

\section{Keywords-IoT, RFID, ESP8266, Image Processing}

\section{INTRODUCTION}

Automation in various fields has evolved to a greater extent over the years. A lot of human efforts have been brought down and different jobs are done with ease. This automation is been found in almost all domains today and it has a greater impact on us on a daily basis. These various technologies are very much required for us to have a quality life. The IoT (Internet of Things) is one of the most widely used technology as it has the ability and boundless potential to do almost everything one could imagine. IoT has evolved from M2M communication, which works with a sensor network and a large number of devices that connect systems, people and other applications to receive and share data through the cloud. These sets of devices together form an ecosystem in which all are interconnected. This IoT is almost implemented in almost all fields like education, agriculture, manufacturing, smart homes etc. Specifically looking into the transportation applications, with the help of mobile and connectivity advancements, the IoT in smart transport has radically changed the transportation industry. The IoT has made everything possible to make trucking a more efficient system - from adequate road safety issues to supply chain management system monitoring. By using IoT, computers have the ability to stay interconnected with mechanical and digital machines. Human -Human or Human - Machine interaction can be reduced to a greater extent by implementing this technology. Different industries and various kinds of organizations due to the implementation of IoT can increase the rate of completion of the work in a short span and reduce manpower. By this, these organizations can operate more efficiently.

\section{LITERATURE SURVEY}

\section{A. Automated Toll Tax Collection System using Cloud}

Database: Etqad Khan et al [1]

This paper proposes an automated toll collection system can be implemented on highways and roads to avoid the hassle and congestion that is caused by vehicles on toll booth that can be mitigated permanently. This uses the technology of RFID where the vehicles can have an uninterrupted ride and the transaction process is smooth. This is one of the IoT applications which can be implemented to reduce the time and manpower at the toll booth to physically collect the money and issue the bills.

The system uses an active RFID tag, which has its battery source and an antenna to communicate with the RFID reader. The tag has to be installed during vehicle registration which will possess a unique ID that will be present in the cloud database [3]. So, when a vehicle comes to the toll plaza, tag communicates with the reader, upon which tag data is verified in the cloud. If the ID matches, the gate opens, else the gates remain closed. A notification regarding the crossing of the gate and the amount deducted is sent on the mobile application, integrated with the cloud.

This system is easy to implement and efficient, which also has a wider range of sensing. It is fast and hence the processing of data is done at a faster rate.

\section{B. IoT based Smart Signal: Shreyas $C N$ et al [3]}

This paper proposes an IoT based automated surveillance system for the vehicles, which captures the registration number of the vehicles and alerts the nearest police stations in case of any pending case, fines or violation of emission norms. Traffic lights are controlled automatically based on live traffic. By implementing this traffic in cities can be easily handled and the vehicles can be monitored. It is also advisable to implement this at toll booths as it can be helpful in tracking stolen vehicles. It is a challenging task to detect the outer boundary of the number plate since the quality of the camera is limited when we take into consideration the various textures styles. Also, the poor quality of light at times makes it difficult to detect the number plate.

The automated surveillance system uses image processing to check the authenticity of the registration plate, fines due or if the vehicle is a stolen one. The extracted plate number is used to search through the RTO database to get detailed information about the vehicle near the signal. In case of any abnormality detected with the vehicle, which alerts a patrol party nearby. A camera is placed at every traffic signal which is used to capture images at a high rate, captured images are then stored in a cloud along with metadata such as the location of the signal from which the image was captured and the time at which the image was captured. Image processing is done using MATLAB and 
Python. Also uses Push Bullet(SMS/Web Service). Cameras can record clear images even at night and also during bad weather conditions, hence making it reliable at all conditions. It also has an algorithm that controls the timer of the signal based on the traffic density.

\section{PROPOSED ARCHITECTURE}

These papers mainly focus on Transportation, which is a necessity for human beings contributing to economic growth. Due to urbanization and other factors, there is an increase in vehicular traffic exponentially, leading to congestion on roads. The papers reviewed to give us certain solutions that mitigate things like traffic, vehicle theft and also creates a smooth journey for vehicles going through toll booths.

Implementation of these mainly would decrease manpower, saves a considerable amount of time and works efficiently. The automatic toll collection system speeds up the toll collection process and the IoT based smart signal helps in managing the traffic signals smartly according to the needs. These devices are prone to various external conditions and hence making it feasible to work in any situation. Despite these advantages, there is a possibility of quite a few disadvantages

The first paper, written by Etqad Khan, Dipesh Garg, Rajeev Tiwari and Shuchi Upadhyay is based on the Automated Toll collection system [1]. This paper proposes the use of RFID tags in vehicles for the automatic toll payment at toll booths on highways. The need for passive tags is necessary because the RFID tag will get energized only when it comes in contact with the reader's magnetic field. The Arduino, interfaced with the ESP8266 Wi-Fi module is connected to the tag. The Arduino is also used in programming the mobile application and is also programmed to accept the authentic RFID tags embedded in the vehicles. The cloud maintains a database of the authentic IDs and when required, will provide the required ID for checking to allow the vehicles to pass through toll gates.

The suggested hardware implements an Arduino Uno, ESP8266 Wi-Fi Module and RFID tags. We know Arduino is a microcontroller unit and ESP8266 is interfaced to provide the Wi-Fi network. We can go for an alternative, combining both microcontroller and Wi-Fi modules together. We can utilize the ESP8266 NodeMCU which consists of an inbuilt TCP/IP stack protocol and can also be used for programming. It has several advantages [4]. It is cost-efficient, has a Flash of $4 \mathrm{MB}$, a clock speed of about 24-52 MHz, more RAM and has an operating voltage of $3.3 \mathrm{~V}$ [3]. It only requires the right kind of RFID tag for interfacing. But on the whole, its functions are pretty similar to the Arduino Uno. It is advisable to use a specific board based on the application. The block diagram with the NodeMCU interfaced can be implemented as shown in fig 1 .

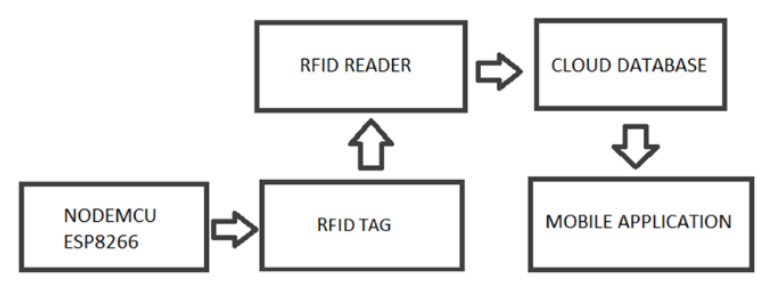

Fig 1: Block Diagram of Proposed Architecture

\section{CONCLUSION}

The Internet of Things guarantees to reshape and improve not only the lifestyle of individuals but also the global market scenario as it increases the gross productivity of an organization. Through a widespread network of smart devices, IoT can encourage plugins and upgrades to vital services in transportation, security, infrastructure, healthcare and welfare, traffic management, etc. whilst also establishing a new application development habitat. Specifically speaking, IoT offers better communication, control and data distribution and decimation at every transportation layer. It stretches across the entire network of all transportation strands like traffic control, parking, fuel consumption, etc. The increasing number of smart devices connected to the web has given IoT a promising future and hence instilling smart technologies in districts all over the world.

\section{ACKNOWLEDGMENT}

We would like to express our deep appreciation and indebtedness to Mr. Santosh for his participation and assistance for the successful completion of this paper.

\section{REFERENCES}

[1] Etqad Khan, Dipesh Garg, Rajeev Tiwari and Shuchi Upadhyay, "Automated Toll Tax Collection System using Cloud Database", 3rd International Conference On Internet of Things: Smart Innovation and Usages (IoT-SIU), February,2018.

[2] Sabbir Ahmed, Tamkin Mahmud Tan, Anna Mary Mondol, Zawad Alam, Noshin Nawal and Jia Uddin, "Automated Toll Collection System Based on RFID Sensor", International Carnahan Conference on Security Technology (ICCST), October,2019

[3] Shreyas C N, Srivatsa V, Vaibhav Pitale and M Ananda, "IoT Based Smart Signal", 2nd International Conference on I-SMAC (IoT in Social, Mobile, Analytics and Cloud) (I-SMAC), August, 2018.

[4] Manish Prakash Gupta, "Google Assistant Controlled Home Automation", International Research Journal of Engineering and Technology (IRJET), Volume: 05 Issue: 05 , May-2018 